\title{
Analysis of Gaps between the Predictive and the Adaptive Component in ATM
}

\author{
Claus Gwiggner and Sakae Nagaoka \\ Electronic Navigation Research Institute, Tokyo, Japan \\ Vu Duong \\ Eurocontrol Experimental Center, Paris, France
}

\begin{abstract}
In a data analysis, we discovered systematic gaps between the number of planned and the number of realized entries of aircraft into flight sectors. We also found that random disturbances of an arrival process into flight sectors cause systematic gaps. We conclude that even if all controllable uncertainties in flow planning were eliminated, systematic gaps between the number of planned and realized traffic would remain. The results give insight into the macroscopic relationship between planned- and realized traffic flows and are a step towards a realistic model of air-traffic flow.
\end{abstract}

\section{Introduction}

The aim of air traffic management (ATM) is to achieve safe, economical and efficient flows of traffic ${ }^{1}$. Major problems with traffic flow are congestion and delays, mainly as a result of an imbalance between capacity and demand ${ }^{2,3}$. ATM consists of the two components flow planning and flow control. The mission of flow planning is to balance airspace demand with available capacity. It is a long-term process, ranging from research on new sector- and route designs (strategic phase) over the analysis of flow patterns (pretactical phase) until the daily schedule of departure slots to avoid capacity violations (tactical phase). Flow planning has to anticipate the future evolution of the network. For this reason, we call it the predictive component in ATM. Once an aircraft is in the air, traffic controllers guide it from origin to destination. Each sector is directly supervised by two controllers, one in charge of conflict detection, and the other in charge of conflict resolution. Minimum distances between aircraft have to be kept. This is achieved by re-routing and speed adjustments. The difference to flow planning is that flow control adapts its behavior to the real traffic situation. For this reason we call it the adaptive component in ATM.

Intuitively, a flow is a number of aircraft per time that move into the same direction. The left part of Figure 1 shows the traffic flows in Central European Airspace, covering the region between London in the north, Zurich in the south and Berlin in the east. The two colors yellow and brown mark the different directions north-south and vice verse. One can see two major traffic flows in north-south direction and a network of remaining flows. The situation in Japan is similar (right part): main flows exist towards the two Tokyo airports Narita and Haneda (yellow, blue), complemented by a large number of additional flows.

Today's flow planning is based on the assumption that aircraft respect their flight plans. But in reality, many factors of uncertainty (e.g. weather conditions, delay from connecting flights, etc.) lead to deviations of aircraft from their flight plans. Ball et al. ${ }^{4}$ classify uncertainty into three categories, shown in Table 1. These categories are 'demand uncertainty', 'capacity uncertainty' and 'flow control uncertainty'. The above examples of uncertainty would fall into the category 'demand uncertainty'.

On a sector level, uncertainties that have not been taken into account during flow planning translate into traffic gaps between the planned number and the realized number of aircraft arriving at sectors. This leads to safety problems (too many aircraft arrive at a sector) and non-optimally used capacity (fewer aircraft than planned arrive at the sector).

In this paper, we show that traffic gaps occur systematically, both, in European and in Japanese Airspace. This is insight into the macroscopic relationship between planned- and realized traffic flows. It is useful 

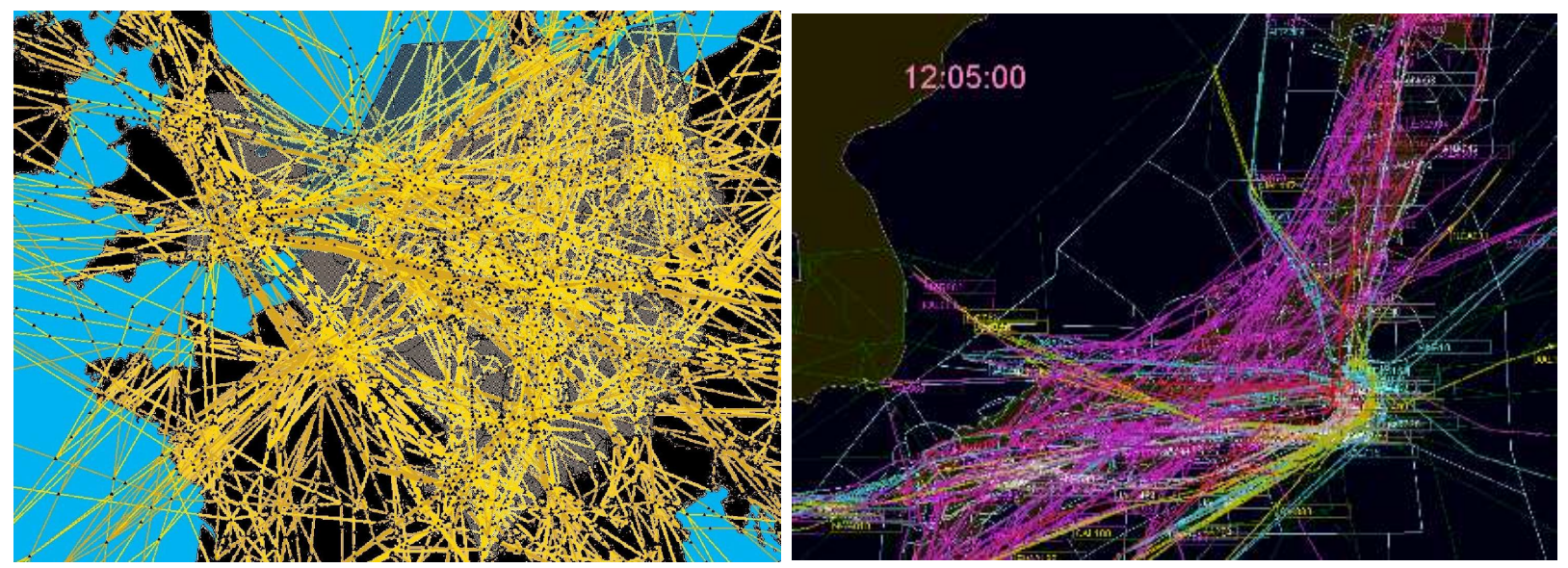

Figure 1. Traffic Flows. Left: European Central Airspace. Right: Japanese Airspace.

\begin{tabular}{|l|l|}
\hline Uncertainty & Factors \\
\hline Demand uncertainty & $\begin{array}{l}\text { flights fail to meet planned departure, ar- } \\
\text { rival or en-route travel times. Contributing } \\
\text { factors are mechanical problems, boarding } \\
\text { passengers or weather conditions. }\end{array}$ \\
\hline Capacity uncertainty & $\begin{array}{l}\text { airport and airspace throughput levels vary. } \\
\text { Contributing factors are weather conditions } \\
\text { and changes in flight sequences that disturb } \\
\text { scheduled departure or arrival spacing. }\end{array}$ \\
\hline $\begin{array}{l}\text { Flow control uncer- } \\
\text { tainty }\end{array}$ & $\begin{array}{l}\text { actions are taken by the traffic controllers } \\
\text { in response to demand and capacity un- } \\
\text { certainty. Examples are re-routing, re- } \\
\text { sectorization and temporary capacity lim- } \\
\text { itations. }\end{array}$ \\
\hline
\end{tabular}

Table 1. Classification of Uncertainty (source: Ball et al. ${ }^{4}$ )

information for flow planning, which can be improved by taking into account the reasons that lead to the gaps. The results in this paper are a summary of our previous research published in ${ }^{5-7}$.

Related work can be found in the analysis of traffic flows. Menon et al. ${ }^{8}$ model en-route air traffic flow inspired by highway traffic. A sector is a one-dimensional volume with in-and out flow. Controllers have the possibility to delay aircraft inside their sectors. Based on this, they analyze for example the impact of control decisions in one sector onto flow patterns in other sectors. Hansen ${ }^{9}$ models arrival flows as deterministic queues, where arrival times are disturbed randomly to take into account network delays and other uncertainty factors. This allows him to quantify delays caused by individual aircraft onto the remaining flow. Wanke et al. ${ }^{10,11}$ analyze the effect of uncertainties in sector demand. They identify binomial- and Poisson distributed counts of aircraft entering flight sectors. They also identify major factors with an influence on these distributions.

The remaining article consists of three parts. In the next part, we analyze past flight data, where we discover the gaps and where we build a descriptive model to quantify them and a probabilistic flow model to explain their main causes. Then, we compare the analysis between European and Japanese data and validate our results. Finally we interpret the results and look out for future research. 


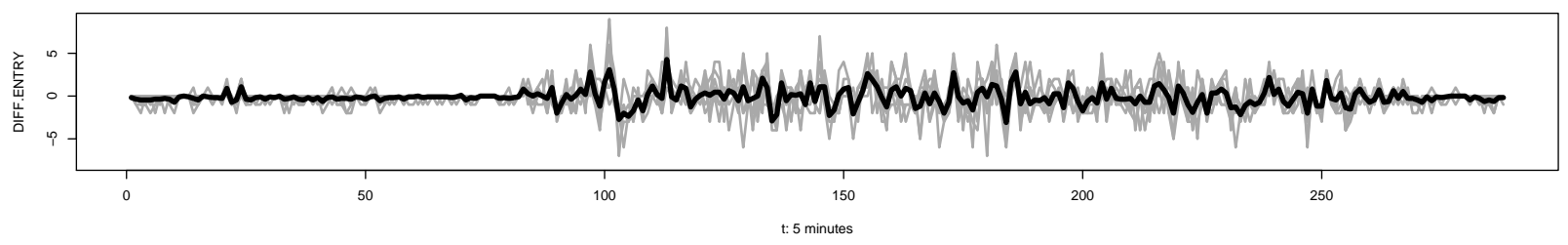

T02 : DIFF.ENTRY

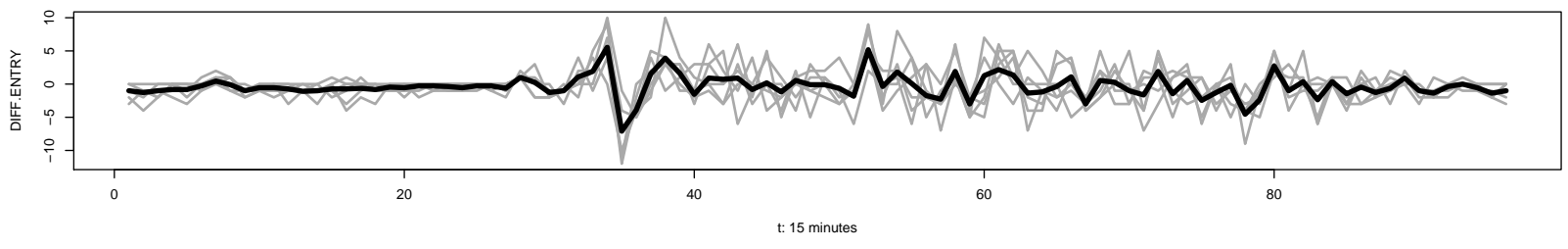

T02 : DIFF.ENTRY

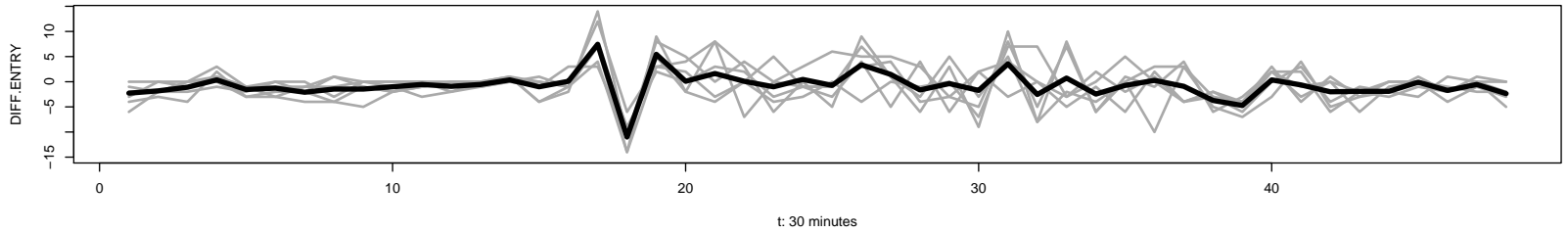

Figure 2. Time plots on different time-scales. Top: $5 \mathrm{~min}$, middle: $15 \mathrm{~min}$, bottom: $30 \mathrm{~min}$ time scale.

\section{Analysis of Gaps}

Gaps are differences between the number of planned and realized entries into a flight sector. They can be represented by three possible definitions

$$
G A P_{t}= \begin{cases}R E A L_{t}-P L N_{t} & \text { absolute } \\ R E A L_{t} / P L N_{t} & \text { relative } \\ f\left(R E A L_{t}, P L N_{t}, \mathbf{X}\right) & \text { functional }\end{cases}
$$

where $R E A L_{t}, t \in \mathbb{N}$ is the number of aircraft that entered a sector in time interval $t$ and $P L N_{t}$ is the number of aircraft that were supposed to enter it. Since the number of real entries into a flight sector is unknown, we consider $G A P_{t}$ as a random process. All three definitions give insight in the phenomenon. For example absolute and relative gaps describe directly how the phenomenon appears to an observer. The functional definition models the joint distribution of $R E A L$ and $P L N . \mathbf{X}$ is a vector describing the environment in which the variables are observed, for example the time of the day. It gives insight into how gaps are generated by the flow planning component.

\section{II.A. Data Analysis}

We use two data sources: flight plan data (before take-off) and radar data. These are trajectory based formats which have to be aggregated on a sector level. All data is numerical. As far as the European data is concerned, we use the aggregation function of the COSAAC ${ }^{12}$ tool (Common Simulator to Assess ATFM Concepts). For the Japanese data, a preprocessing step had to be done in order to interpolate the intersection of the trajectories with the 3-dimensional sector boundaries. Due to these calculations, and general flight-plan inconsistencies (e.g. missing segments), we expect $15 \%$ of inaccurate sector entry times (please see Ref. 7 for more details). Flight plan and radar data are available for 31 en-route sectors on 75 week days (Mon-Thu) in the year 2004 summer period May 13 - Sept. 29 for the European Airspace and for 21 sectors on 6 weekdays in the year 2006 (August and November) for the Japanese Airspace. We 
T01

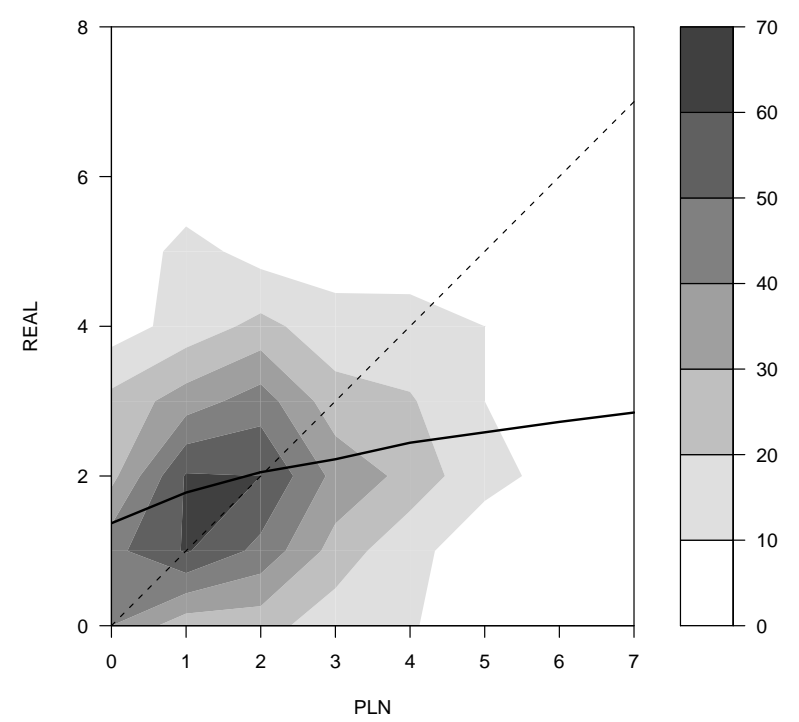

Figure 3. Relationship between number of planned arrivals $P L N$ (x-axis) and average number of real arrivals $R E A L$ (y axis). Distribution and sample means (bold line).

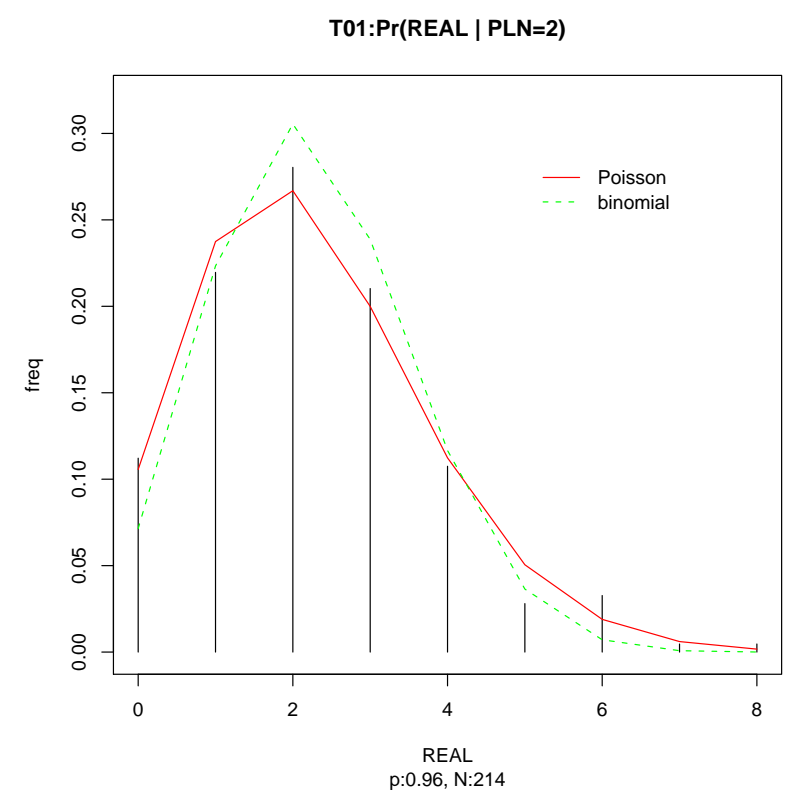

Figure 4. Number of real entries $\left(R E A L_{t}\right)$ conditioned on number of planned entries $\left(P L N_{t}=2\right)$.

assume similar traffic conditions (number of flights, network topology, controllers) for each of these days (more details can be found in References ${ }^{6,7}$ ).

Figure 2 shows the process $G A P_{t}=P L N_{t}-R E A L_{t}$ for a typical Japanese sector on three different time-scales. The timescales grow from $5 \mathrm{~min}$ (upper panel), over $15 \mathrm{~min}$ (middle) to $30 \mathrm{~min}$ (bottom). On the x-axes, the slot numbers are drawn. The upper panel has 288 slots (12 per hour), the middle one 96 (4 per hour) and the lower panel has 48 slots (2 per hour of the day). In each panel, 6 grey lines are superposed, one from each of the available days. The black line is the average over these 6 days. The idea of this plot is to identify daily repeating patterns. For example, at $t=9: 00$ (slot 36 and 18), a negative peak of value -10 


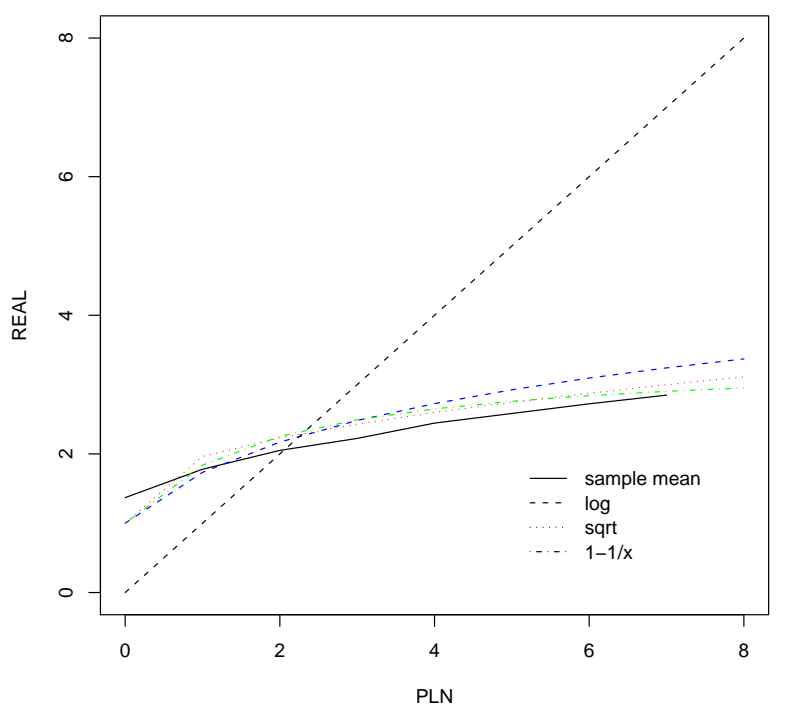

Figure 5. Candidate trend functions. x-axis: number of planned arrivals, y axis: average number of real arrivals.

can be seen. In the remaining time, the process fluctuates around an average value of 0 . Indeed, a one-sided t-test agrees that this mean value is lower than a randomly selected one from the remaining process. But such strong patterns are the exception and not the rule. We could find them in only a few sectors, and in most of the cases, it could not be excluded that they resulted simply from data inaccuracies ${ }^{7}$. We also analyzed the distributions of the processes. Mean and variance of the fluctuation look constant during the day hours (7:00-19:00). For example, the distributions for the data in Figure 2 during the day hours are symmetric, around 0, with standard deviations suggesting that in the majority of the cases, $\pm 25 \%$ of the planned traffic arrives at the sector. Here again, no unexpected patterns could be found on a system level ${ }^{6,7}$.

What was unexpected can be seen in Figure 3. It plots the number of planned entries $\left(P L N_{t}\right)$ on the $\mathrm{x}$-axis against the number of real entries $\left(R E A L_{t}\right)$ on the $\mathrm{y}$-axis. The range of both variables is around $[0,8]$ on a $5 \mathrm{~min}$ timescale. Since there are $N=870$ points in the sample, but only 81 positions they can take, we visualize the distribution of the cloud by the background color (light: few values, dim: many values). One can see a single peak around $(1,2)$ and that the cloud is distributed symmetrically around this peak. Negative values seem to be 'cut-off'. Figure 4 shows a typical distribution of a column in this plot $P\left(R E A L_{t} \mid P L N_{t}=k\right)$. On the x-axis, we see the number of aircraft that enters a sector, on the y-axis the frequency of such events. In the example $P L N_{t}=2$, which is the average in-flow for this sector. The distribution is right skewed. The variable is positive (including 0) and discrete. Superposed are a Poisson (red) and a binomial distribution (green, dotted). Both distributions are accepted by a $\chi^{2}$ goodness-of-fit test (on a $5 \%$ level). A system-wide comparison showed that $70 \%$ of such column distributions can be seen as Poisson and $26 \%$ as binomial ${ }^{6,7}$.

Going back to Figure 3, the diagonal (dotted line) corresponds to the case of perfect planning: exactly the same number of aircraft than planned arrive in reality. Due to uncertainties, one expects a fluctuation around this line. But this is not the case. The sample average is the black line. It corresponds to the points:

$$
\hat{\mu}\left(R E A L_{t} \mid P L N_{t}=k\right)=\frac{1}{n_{k}} \sum_{P L N_{i}=k} \text { real }_{i}
$$

where $n_{k}$ is the number of observations with $P L N_{t}=k$. As a function of $k$, it has a logarithm-like shape (bold line). In particular at $P L N_{t}=0$, the mean is $>0$. Figure 5 shows this mean line in more detail. We model it as a function of the planned number of aircraft: $R E A L_{t}=f\left(P L N_{t}\right)$. Three candidate functions are a logarithmic (blue), a square-root (red) and a reciprocal one (green). They all describe accurately the sample data (black). 


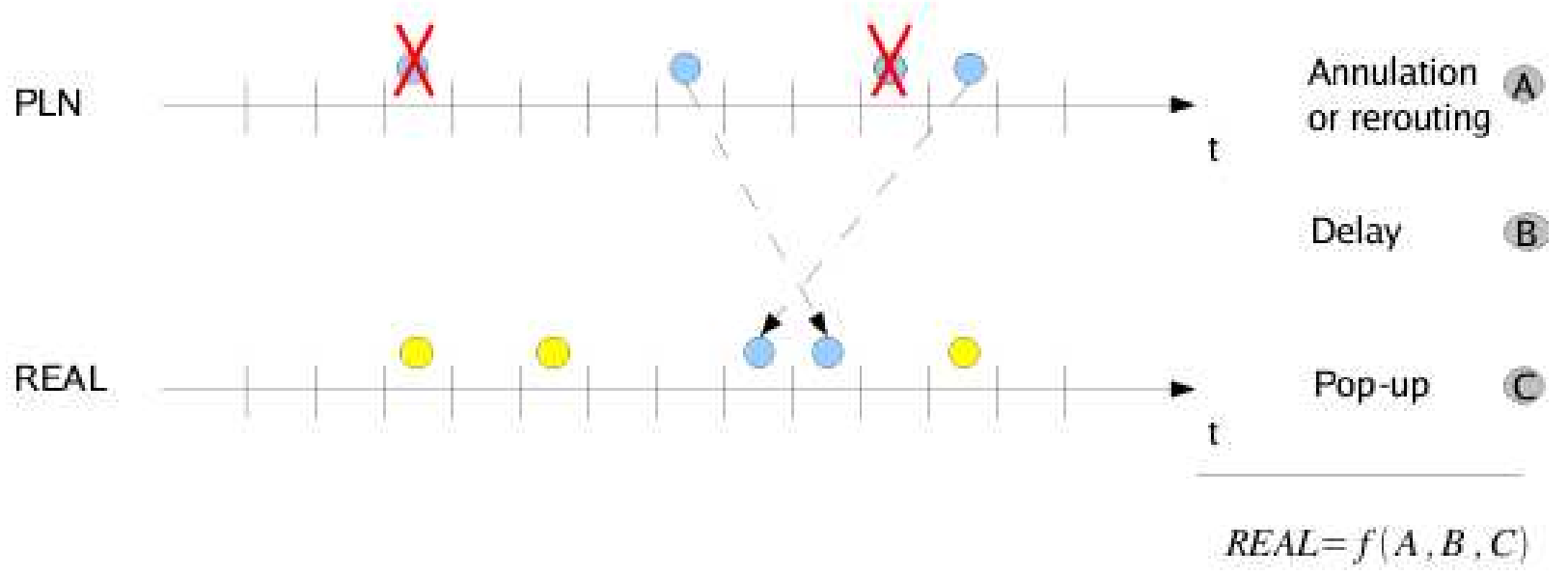

Figure 6. Events at a sector entry.

To summarize, we have seen the uncertainty of traffic gaps (Figures 3 and 4) and their average behavior (Figure 5). Combining this, we are able to pose the following descriptive model of the traffic gaps:

$$
\begin{aligned}
P(R E A L=n \mid P L N=k) & =\frac{e^{-\mu_{k}} \mu_{k}^{n}}{n !} \\
\log \left(\mu_{k}\right) & =\alpha f(k)+\beta
\end{aligned}
$$

with $\alpha, \beta \in \mathbb{R}$ and $f(k) \in\{\log (k), \sqrt{k}, 1-1 / k\}$. We fit the above models by maximum likelihood, using a Newton-Raphson based iterative method ${ }^{13}$. These are linear counting models, which have always a unique solution and positive values for the mean parameter ${ }^{14}$. Note that the model using the logarithmic trend can also be written as the power of $\alpha$. Our Goodness-of-fit analysis showed that (i) the models explain a large part of the variation in the data and (ii) the different candidate trend functions $f$ explain equally well the data $^{6}$.

\section{II.B. Probabilistic Analysis}

While the previous analysis allows to describe the traffic gaps, it cannot explain why they occur. It is natural to think that random events deviate flights from their flight plans. For example, weather conditions or unpredictable events (e.g. passenger delay or technical failure) constantly affect the system. One likes to assume that such events do not disturb the flow planning systematically. In average, their effects should be canceled out. On the other hand there are mechanisms that systematically disturb the flight plans. For example, airlines use the cancellation of flights as a strategy to avoid high departure delays ${ }^{15}$.

As in Wanke et al. ${ }^{16}$ or Ball et al. ${ }^{4}$ we distinguish three types of events that disturb a flight planning (Figure 6):

- Cancellation and rerouting: some of the scheduled flights are canceled or rerouted to other sectors.

- Delay: aircraft arrive too early or too late at the sector. Delay occurs either at the departure airport or during the flight.

- Pop-up: rerouted aircraft from other sectors or aircraft without submitted flight plans arrive. 


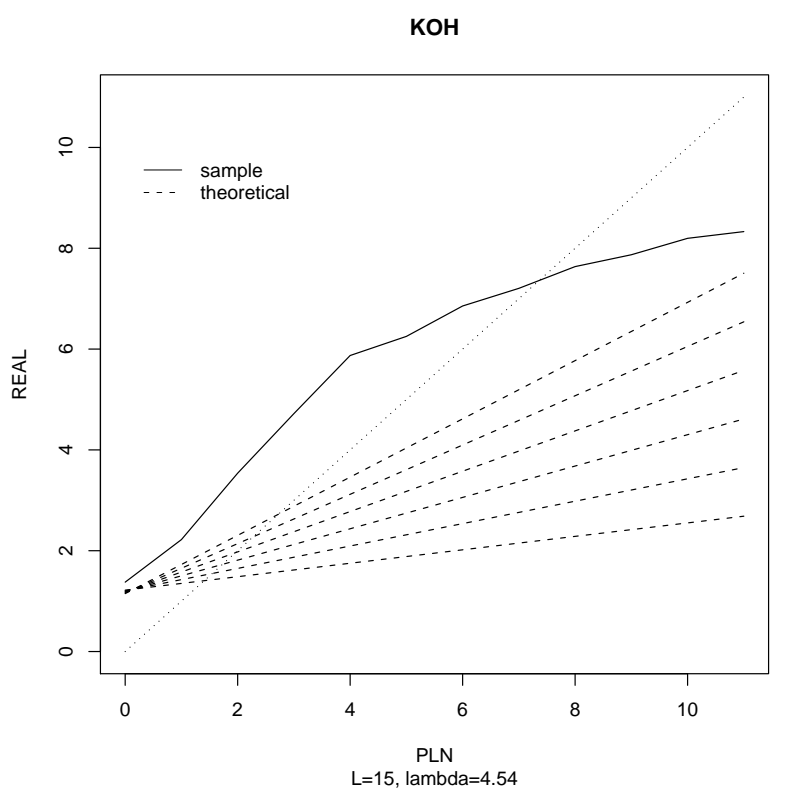

Figure 7. Conditional expectations of randomly translated Poisson process.

We formalized this situation by thinking of an arrival process at a sector as a randomly translated Point process ${ }^{17}$. In this paper we only summarize our results. Please consult Ref. 6 for a mathematical discussion. For a given sector, we analyzed

$$
\left\{\tau_{i}\right\} \rightarrow\left\{\tau_{i}+\epsilon_{i}\right\}
$$

where $\tau_{i} \in \mathbb{R}$ is the planned arrival time of aircraft $i$ and $\epsilon_{i}$ is a random error for the arrival.

Figure 7 plots $P L N_{t}$ against $R E A L_{t}$ as before. The diagonal corresponds to perfect planning, where exactly the same number of aircraft arrives than has been planned. The sample averages (bold curve) are obtained from the data (for a European sector in this case, with $\mathrm{L}=15 \mathrm{~min}$ time-slots). What is new in this plot are the dotted lines. They are the average traffic gaps based on our model. This model assumes that $\left\{\tau_{i}\right\}$ is a Poisson process with arrival rate $\lambda$ and that $\epsilon$ is a random variable with mean $\mu$ and variance $\sigma^{2}$, where $\epsilon_{i}, \epsilon_{j}$ are uncorrelated for $i \neq j$. From this we calculated the distribution of the number of aircraft entering a sector given that a number $k$ had been planned to enter it. After scaling the variance appropriately, it turned out that the mean value of this distribution is a linear function of the traffic flow: $\mu_{k} \sim\left(1-\dot{\sigma}^{2}\right) k+\dot{\sigma}^{2} \lambda$. The slope of this function decreases with the scaled variance $\dot{\sigma}^{2}$ of the disturbances: the higher this variance, the larger the gaps between planned and realized sector entries. The intercept is proportional to the arrival rate $\lambda$ and the scaled variance $\dot{\sigma}^{2}$ of the disturbances. This means that systematic traffic gaps occur, even if the only force on the system is a random disturbance of the arrival times. And this independently of the distribution type of the disturbances (it is only dependence on its mean and variance). Finally, since homogeneous Poisson arrivals are unrealistic, we simulated the effect of random disturbances on the (deterministic) arrival processes, obtained from the flight plan data. In this case the mean values showed a logarithmic shape, similar to the sample data. But they did not fit with the sample data. This means that in reality, the disturbances are not additive or independent and identical from each other.

In summary, we found (i) that random disturbances of flight plans lead to systematic gaps between the number of planned and the number of realized sector entries. And (ii), the data shows that pure randomness is not the only disturbance factor.

\section{Comparison between Japanese and European Flow Data and Validation}

Comparing two ATM systems, as done for example between U.S. and Europe ${ }^{18}$ is beyond the scope of this section. We only address some similarities about the performance of flow planning in the European and the Japanese ATM that we detected in our data. The two systems share some characteristics, for example 


\begin{tabular}{|c|c||c|}
\hline Technique & Attribute & Comparison \\
\hline Time plot & $\begin{array}{c}\text { Mean } \\
\text { Variance }\end{array}$ & similar \\
& similar \\
\hline Distribution & Core & similar \\
$($ abs, rel $)$ & Mean & similar \\
$\ldots \ldots \ldots \ldots \ldots$ & $\ldots \ldots \ldots \ldots$ & $\ldots \ldots \ldots \ldots$. \\
& Variance & differ \\
& Tail & differ \\
$\ldots \ldots . . . \ldots$ & $\ldots \ldots \ldots .$. & $\ldots \ldots \ldots \ldots$ \\
(cond) & Poisson & similar \\
\hline Trend & Log-like & similar \\
\hline Propagation & Time & similar \\
& Space & similar \\
\hline
\end{tabular}

Table 2. Comparison Japanese- and European Airspace Data.

similar procedures, mechanisms and equipment. Difference between them are for example the major flow patterns, or the cultural context. For example, it could be interesting to know if European and Japanese controllers treat stressful situations differently.

In our data analysis, we compared 10 different characteristics, as shown in Table 2. For example, the core-parts of the distributions ( $3^{\text {rd }}$ row) turned out to be similar in European and Japanese sector data. Or, the logarithm-like traffic gaps (row 8) also occurred in both data sets. The only differences we found were small ones in the variance and the tail behavior of the distributions (rows 5 and 6 ). For example traffic gap distributions in European Airspace show higher tails than those in Japanese Airspace, suggesting that very large gaps occur more frequently in European Airspace than in Japanese. ${ }^{7}$. We concluded that all main characteristics were similar in both European and Japanese data. But a comparative analysis with mature methodology is needed to confirm these findings.

Besides this, we validated all our findings on all analyzed sectors (31 sectors in European, 21 sectors in Japanese Airspace) and on three different time-scales (5 min, $15 \mathrm{~min}$ and $60 \mathrm{~min}$ ). Please $\mathrm{see}^{5-7}$ for the details.

\section{Conclusions}

We analyzed gaps between the planned and the realized number of aircraft entering flight sectors. We called these gaps 'traffic gaps' between the predictive and the adaptive component in ATM. Our hypothesis was that due to operational uncertainties (e.g. delays, technical failures, etc.), there are systematic gaps between these two numbers. We proposed a descriptive model of traffic gaps to quantify them and a probabilistic model to show that randomly disturbed flight schedules already lead to systematic traffic gaps.

From our analysis we conclude that in both, European and Japanese Airspace, (i) gaps between the number of planned and realized traffic occur systematically, and this (ii) even if the only force on the system are random disturbances of arrival times. This is counter-intuitive since one expects that different uncertainty factors cancel out in average. Such information is useful to improve flow planning because they give insight into the macroscopic relationship between planned- and realized traffic flows.

As future work we propose to analyze statistical models that explain the dynamics of traffic flows and their congestion patterns.

\section{Acknowledgments}

The first author likes to thank to M. Pierre Collet and M. James K. Lindsey for discussions on the topic. 


\section{References}

${ }^{1}$ ICAO. Global Air Traffic Management Operational Concept. International Civil Aviation Organization, 2005.

${ }^{2}$ M. Ball, C. Barnhart, G. Nemhauser, and A Odoni. Handbooks in Operations Research 8 Management Science: Transportation Volume 14. Gilbert Laporte and Cynthia Barnhart (editors), chapter 1, pages 1-68. Elsevier, 2007.

${ }^{3}$ M. Janic. Air Transport System Analysis and Modelling: Capacity, Quality of Services and Economics. Gordon and Breach Science Publishers, Amsterdam, 2000.

${ }^{4}$ M.O. Ball, D. Lovell, R. Hoffman, and A. Mukherjee. Response mechanisms for dynamic air traffic flow management. In Proceedings of the 6th Europe-USA ATM Seminar. Baltimore. US., 2005.

${ }^{5}$ C. Gwiggner and V. Duong. Averages, Uncertainties and Interpretation in Flow Planning. In Proceedings of the 2nd International Conference on Research in Air Transportation. ICRAT 2006, Belgrad, Serbia, 2006.

${ }^{6}$ C. Gwiggner. Analyse des incertitudes dans les flux du trafic aérien. PhD Thesis. Ecole Polytechnique. France, 2007.

${ }^{7}$ C. Gwiggner, K. Kageyama, and S. Nagaoka. Common Trends in Japanese- and European Airspace Data. Technical report, ENRI Kenkyu-Happo Kay Presentation, 2008.

${ }^{8}$ P. K. Menon, G. D. Sweriduk, and K. Bilimoria. New approach for modeling, analysis and control of air traffic flow. In AIAA Journal of Guidance, Control and Dynamics, volume 27/5, pages 737-744, 2004.

${ }^{9} \mathrm{M}$. Hansen. Micro-level analysis of airport delay externalities using deterministic queuing models: a case study. Journal of Air Transport Management, 8-2:73-87, 2002.

${ }^{10}$ C. Wanke, M.B. Callaham, D.P. Greenbaum, and A.J. Masalonis. Measuring uncertainty in airspace demand predictions for traffic flow management applications. AIAA Guidance, Navigation and Control Conference. Austin, TX, USA., 2003.

${ }^{11}$ C. Wanke, L. Song, S. Zobell, D. Greenbaum, and S. Mulgund. Probabilistic congestion management. Proceedings of the 6th Europe-USA ATM Seminar. Baltimore. US., 2005.

${ }^{12}$ EUROCONTROL. Cosaac User Manual. Release 3.9.3. Technical report, EEC, 2004.

${ }^{13} \mathrm{R}$ Development Core Team. R: A Language and Environment for Statistical Computing. R Foundation for Statistical Computing, Vienna, Austria, 2008. ISBN 3-900051-07-0.

${ }^{14}$ J.K. Lindsey. Modelling Frequency and Count Data. Oxford University Press, 1995.

${ }^{15}$ A. Mukherjee, D. Lovell, M. Ball, A. Odoni, and G. Zerbib. Modeling delays and cancellation probabilities to support strategic simulations. In Proceedings of the 6th Europe-USA ATM Seminar. Baltimore. US., 2005.

${ }^{16} \mathrm{C}$. Wanke, S. Mulgund, D. Greenbaum, and L. Song. Modeling traffic prediction uncertainty for traffic management decision support. AIAA Guidance, Navigation and Control Conference. Providence RI, 2004.

${ }^{17}$ D.R. Cox and V. Isham. Point Processes. Chapman and Hall, 1980.

${ }^{18}$ EUROCONTROL. A comparison of performance in selected US and European En-route Centres. EUROCONTROL, 2003. 